International Journal of Pure and Applied Mathematics

Volume 89 No. 3 2013, 323-334

ISSN: 1311-8080 (printed version); ISSN: 1314-3395 (on-line version)

url: http://www.ijpam.eu

doi: http://dx.doi.org/10.12732/ijpam.v89i3.3

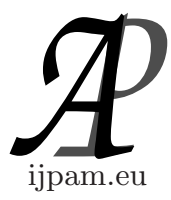

\title{
FUZZY GOAL PROGRAMMING BASED ON PIECEWISE LINEAR MEMBERSHIP FUNCTIONS
}

\author{
Ksh. Mangijaobi Devi ${ }^{1}$, Th. Biren Singh ${ }^{2}$ \\ ${ }^{1}$ Department of Mathematics \\ Manipur University \\ Imphal, 795003, Manipur, INDIA \\ ${ }^{2}$ Oriental College \\ Imphal, 795001, INDIA
}

\begin{abstract}
In the paper, we propose a method for finding the solution of fuzzy linear goal programming(FLGP) problems where each objective function has a prescribed fuzzy goal characterized by piecewise linear membership functions. In view of the fuzzy membership functions and the given constraints, we employ deviational variables and then the problem is transformed into a classical linear programming(LP) problem. The proposed method is mainly based on the concepts of piecewise linear functions due to Charnes and Cooper [2] and Can and Houk [5]. An example is illustrated to show the more applicability of the proposed method.
\end{abstract}

AMS Subject Classification: 03B52, 90C29

Key Words: fuzzy goal programming, piecewise linearmembership functions, fuzzy environment

\section{Introduction}

Initially introduced as an application of fuzzy set theory to goal programming

Received: April 1, 2013

(C) 2013 Academic Publications, Ltd.

${ }^{\S}$ Correspondence author url: www.acadpubl.eu 
by Narasimhan [13] and Hannan [6], fuzzy goal programming(FGP) gained popularity in the area of multiple criteria optimization problems under fuzzy environment. Tiwari et al [14] investigated how pre-emptive(lexicographic) priority structure could be used in FGP problems. In [15] an additive model for FGP problems has been introduced. Flavell [11] investigated a new goal programming formulation.Lohgaonkar et al [10] introduced an additive fuzzy multiple goal programming model for unbalanced multi-objective transportation problem. An interactive fuzzy goal programming for multi-objective transportation problems has been introduced by Wahed and Lee [17]. Moreover, we can mention the work by [4].

In the paper, we introduce a brief survey of FGP problems in Multi-objective linear programming (MOLP) problems so as to establish a relationship between different approaches under fuzzy environment.

The paper is organised as follows: In Section 2, we present the concepts of fuzzy decision and fuzzy goal programming (FGP) problems. In Section 3,we briefly review some concepts of classical goal programming (GP) problems and the piecewise linear functions due to Charnes and Cooper [2] and Can and Houk [5]. In Section 4, we propose the solution method for solving goal programming problem under fuzzy environment. An example is also illustrated to show the efficiency of the method.

\section{Prerequisite: Fuzzy Decision of Bellman and Zadeh, see [12]}

Let $\mathrm{X}$ be a given set of all possible solutions to a decision problem. A fuzzy goal $\tilde{G}$ is a fuzzy set on $\mathrm{X}$ characterize by its membership function

$$
\mu_{\tilde{G}}: X \rightarrow[0,1] .
$$

A fuzzy constraint $\tilde{C}$ is a fuzzy set on $\mathrm{X}$ characterize by its membership function

$$
\mu_{\tilde{C}}: X \rightarrow[0,1] .
$$

Let $\mu_{\tilde{C}_{i}}(x), i=1,2, \ldots, m$ where $x \in X$ be membership functions of constraints, defining the decision space and $\mu_{\tilde{G}_{j}}(x), j=1,2, \ldots, n, x \in X$ the membership functions of objective(utility) functions or goals.

A fuzzy decision is then defined by its membership function (see Zimmermann [7]):

$$
\mu_{\tilde{D}}(x)=\circledast_{i} \mu_{\tilde{C}_{i}} * \circledast_{j} \mu_{\tilde{G}_{j}}(x), \quad i=1,2, \ldots, m, j=1,2, \ldots, n,
$$


where $*, \circledast_{i}$ and $\circledast_{j}$ denote possibly, appropriate context-dependent aggregators (connectives).

\section{Model of the Fuzzy Goal Programming Function}

The idea of GP is to establish a goal level of achievement of each criterion. For conventional treatment of goal programming, the reader is referred to sources such as Charnes and Cooper [1], Lee [16], Ignizio [8]. In this section, we considered a more critical look at fuzzy goal programming originated from MOLP problems.

A classical structure of multi-objective linear programming model with $\mathrm{k}$ linear objective functions is as follows:

$$
\begin{gathered}
\text { Minimize } z(x)=\left(z_{1}(x), z_{2}(x), \ldots, z_{k}(x)\right), \\
\text { subject to } A x \leq b, \\
x \geq 0,
\end{gathered}
$$

where

$$
z_{i}(x)=\sum_{j=1}^{n} c_{i j} x_{j},
$$

$c_{i}=\left(c_{i 1}, c_{i 2}, \ldots, c_{i n}\right), i=1,2, \ldots, k, x=\left(x_{1}, x_{2}, \ldots, x_{n}\right)^{T}, b=\left(b_{1}, b_{2}, \ldots, b_{m}\right)^{T}, A$ is $m \times n$ matrix and $T$ denotes the transpose of a vector.

Assume that the decision maker(DM) proposes fuzzy goals such as the objective function $z_{i}(x)$ should be substantially less than or equal to some goal value $G_{i}$.In addition to above assumption, if it is possible to consider the right hand side(RHS) values of $b$ as a fuzzy resource, then model(2.4) can be written as:

Find $x$

$$
\begin{gathered}
\text { subject to } z(x) \cong \tilde{G}, \\
A x \leq \tilde{b}, \\
x \geq 0 .
\end{gathered}
$$

Let the membership function of fuzzy goal $\tilde{G}, \mu_{\tilde{G}}(z(x))$ be $\mu_{\tilde{G}}: X \rightarrow[0,1]$.

By using the fuzzy decision, "max-min" of Bellman and Zadeh [12] and by introducing the auxiliary variable $\alpha, \alpha \in[0,1]$,a FGP problem adopts the following formation: 


$$
\begin{gathered}
\text { subject to } \alpha \leq \mu_{\tilde{G}}(z(x)), \quad i=1,2, \ldots, k, \\
A x \leq \tilde{b}, \\
x \geq 0 .
\end{gathered}
$$

\section{The Concept of Classical Goal Programming}

The traditional form of a goal programming (GP) problem with one of each type of goal criterion is expressed as

$$
\begin{gathered}
\operatorname{goal}\left\{f_{i}(x)=z_{i}\right\}, z_{i} \in\left[z_{i} \geq t_{i}, z_{i} \leq t_{i}, z_{i}=t_{i}, z_{i} \in\left[t_{i}^{l}, t_{i}^{u}\right]\right], \quad i=\{1,2, \ldots, k\}, \\
\text { subject to } x \in F, x \geq 0 .
\end{gathered}
$$

where $t_{i}$ is target level for the $i^{t h}$ goal,$x \in R^{n}$ is vector of decision variables, $\mathrm{F}$ is feasible set of constraints, $t_{i}^{l}$ and $t_{i}^{u}$ are the lower and upper limits of $z_{i}$ respectively.

A key element of GP model (3.1) is the achievement function that represents a mathematical expression of unwanted deviation variables. The three existing forms of GP achievement functions are (i) weighted GP(WGP), also known as Archimedean GP, (ii)lexicographic GP(LGP), also known as non-Archimedean or pre-emptive GP, and (iii) MINMAX GP(MGP), also known as Fuzzy programming or Chebyshev. The analytical structure of a WGP model of model (3.1) is expressed following Ignizio [8]:

Achievement function:

$$
\operatorname{Min} \sum_{i=1}^{k}\left(\alpha_{i} n_{i}+\beta_{i} p_{i}\right) .
$$

Goals and constraints:

$$
\begin{gathered}
f_{i}(x)+n_{i}-p_{i}=t_{i}, \quad i=\{1,2, \ldots, k\}, \\
x \in F, \quad x \geq 0, \quad p \geq 0, \quad n \geq 0,
\end{gathered}
$$

where $n_{i}, p_{i}$ are negative and positive deviations from the target value $t_{i}$ of $i^{\text {th }}$ goal, $\alpha_{i}=w_{i} / q_{i}$ if $n_{i}$ is unwanted, otherwise $\alpha_{i}=0, \beta_{i}=w_{i} / q_{i}$ if $p_{i}$ is unwanted, otherwise $\beta_{i}=0, w_{i}$ and $q_{i}$ are the weights reflecting preferential and normalising purposes attached to the achievement of the $i^{t h}$ goal. 
The solution of WGP provides the maximum aggregated achievement between the different goals (see e.g. Dyer [9]). The analytical structure of a LGP is the following (Ignizio [8]).

Achievement function:

Lex $\min \left[a=\sum_{i \in h_{1}}\left(\alpha_{i} n_{i}+\beta_{i} p_{i}\right), \ldots, \sum_{i \in h_{r}}\left(\alpha_{i} n_{i}+\beta_{i} p_{i}\right), \ldots, \sum_{i \in h_{Q}}\left(\alpha_{i} n_{i}+\beta_{i} p_{i}\right)\right]$

Goals and constraints:

$$
\begin{gathered}
f_{i}(x)+n_{i}-p_{i}=t_{i}, \quad i \in\{1,2, \ldots, k\}, \\
i \in h_{r}, \quad r \in\{1,2, \ldots, Q\} \\
x \in F, x \geq 0, p \geq 0, n \geq 0,
\end{gathered}
$$

where $h_{r}$ denotes the index set of goals placed in the $r$-th priority level.

It is noted that in Lexicographic achievement functions, there is no finite trade-offs among goals placed in different priority levels (see. e.g. Romero [3]).The analytical structure of MGP model is expressed as Flavell [11]:

Achievement function:

\section{Min D}

Goals and constraints:

$$
\begin{gathered}
\left(\alpha_{i} n_{i}+\beta_{i} p_{i}\right)-D \leq 0, \\
f_{i}(x)+n_{i}-p_{i}=t_{i}, \quad i \in\{1,2, \ldots, k\}, \\
x \in F, x \geq 0, p \geq 0, n \geq 0,
\end{gathered}
$$

where $\mathrm{D}$ is the maximum deviation from any single goal.

MGP is the optimization of a utility function where the maximum deviation is minimized. Thus, this type of achievement function provides a solution that gives the maximum importance to the goal most displaced with respect to its target. An extensive analysis of MGP can be found in [3], [4]. Following Romero [4], other newer versions of formulation with achievement functions that generalise the three classic forms previously introduced are obtained as shown below:

(i) Achievement function: Lex min $a=\left[D_{1}, D_{2}, \ldots, D_{Q}\right]$.

Goals and constraints:

$$
\left(\alpha_{i} n_{i}+\beta_{i} p_{i}\right)-D_{r} \leq 0, \quad i \in h_{r}, r \in\{1,2, \ldots, Q\},
$$




$$
\begin{gathered}
f_{i}(x)+n_{i}-p_{i}=t_{i}, \quad i \in\{1,2, \ldots, k\}, \\
x \in F, x \geq 0, p \geq 0, n \geq 0 .
\end{gathered}
$$

(ii) Achievement function:

$$
\operatorname{Min}(1-\lambda) D+\lambda\left(\alpha_{i} n_{i}+\beta_{i} p_{i}\right) \text {. }
$$

Goals and constraints:

$$
\begin{gathered}
\left(\alpha_{i} n_{i}+\beta_{i} p_{i}\right)-D \leq 0, \\
f_{i}(x)+n_{i}-p_{i}=t_{i}, \quad i \in\{1,2, \ldots, k\}, \\
x \in F, p \geq 0, n \geq 0, \lambda \in[0,1] .
\end{gathered}
$$

(iii)Achievement function:

$$
\begin{gathered}
\text { Lex } \min a=\left[\left(1-\lambda_{1}\right) D_{1}+\lambda_{1} \sum_{i \in h_{1}}\left(\alpha_{i} n_{i}+\beta_{i} p_{i}\right), \ldots,\left(1-\lambda_{r}\right) D_{r}+\right. \\
\lambda_{r} \sum_{i \in h_{r}}\left(\alpha_{i} n_{i}+\beta_{i} p_{i}\right), \ldots,\left(1-\lambda_{Q}\right) D_{Q}+\lambda_{Q} \sum_{i \in h_{Q}}\left(\alpha_{i} n_{i}+\beta_{i} p_{i}\right)
\end{gathered}
$$

Goals and constraints:

$$
\begin{gathered}
\left(\alpha_{i} n_{i}+\beta_{i} p_{i}\right)-D_{r} \leq 0 \\
f_{i}(x)+n_{i}-p_{i}=t_{i}, \quad i \in\{1,2, \ldots, k\}, \\
x \in F, p \geq 0, n \geq 0, \lambda_{r} \in[0,1] .
\end{gathered}
$$

In all the achievement functions presented above two basic assumptions underlie: (1) the decision maker(DM) determines a precise target for each attribute and (2) the deviations regarding the target are penalized with a constant weight independently of which is the distance from the target. With these assumptions we have a piecewise linear penalty function. Charnes et al [2] introduced a piecewise linear penalty function for GP in the following way (Fig $3.1)$.

$$
f(z)=\sum_{j=1}^{N} \alpha_{j}\left|z-t_{i}\right|+\beta z+\gamma
$$




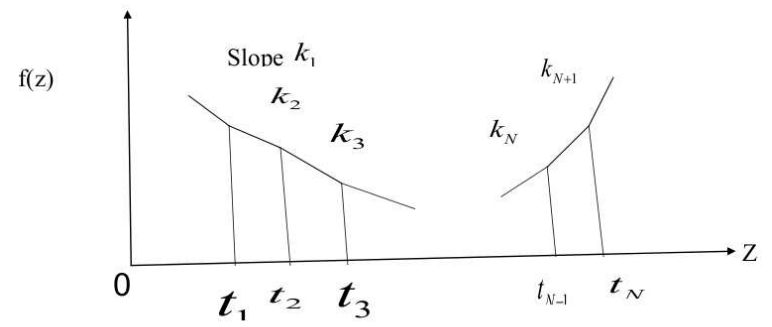

Fig 3.1

where

$$
\alpha_{j}=\frac{k_{j+1}-k_{j}}{2}, \beta=\frac{k_{N+1}+k_{1}}{2}, \gamma=\frac{a_{N+1}+a_{1}}{2},
$$

$j=1,2, \ldots, N, k_{j}$ is the slope, $a_{j}=f(z)$ - intercept, which is a constant, of the line segment initiated at $t_{j-1}$ and terminated at $t_{j}$.

Assuming (3.8), Charnes and Cooper [2] showed how a GP with piecewise linear penalty functions can be formulated as a LP problem. Can and Houk [5] introduced a U-shaped piecewise linear penalty function, like those with five sides(Fig 3.2)

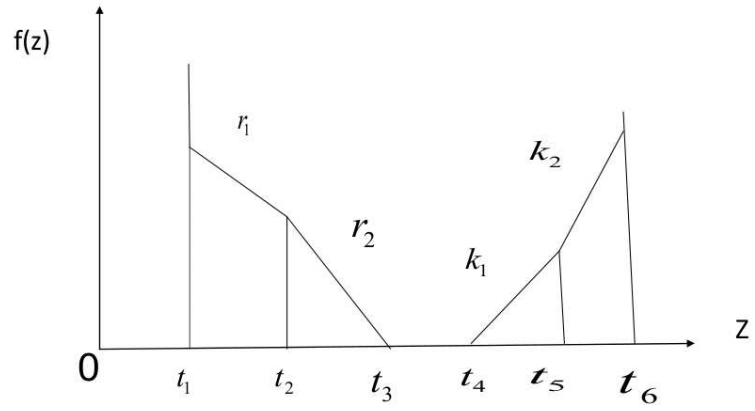

Fig. 3.2, U- Shaped penalty function(five sides)

The penalty function can be represented in the following way:

$$
f(z)=\left|r_{1}\right| n_{1}+\left|r_{2}\right| n_{2}+\left|k_{1}\right| p_{1}+\left|k_{2}\right| p_{2},
$$

where

$$
\begin{gathered}
z+n_{1}+n_{2}+n_{3}-p_{1}-p_{2}=t_{4}, \\
n_{1} \leq t_{2}-t_{1}, n_{2} \leq t_{3}-t_{2}, n_{3} \leq t_{4}-t_{3}, \\
p_{1} \leq t_{5}-t_{4}, p_{2} \leq t_{6}-t_{5},
\end{gathered}
$$


$n, p \geq 0$.

Obviously, the above expression can be generalised in a straightforward manner for penalty functions with $n$ sides.

\section{Linear GP Problem under Fuzzy Environment}

In this section, we propose a method for solving GP problem under fuzzy environment. Assume that we have a Linear GP problem as defined in problem (3.1).

Assume that the DM proposes fuzzy goals such as the objective function $z_{i}(x)$ should be substantially greater than or equal to some value $t_{i}$, $\mathrm{i}=1,2, \ldots, \mathrm{k})$. Then the corresponding fuzzy membership function:

$$
\mu_{i}\left(z_{i}(x)\right), \mu_{i}: R \rightarrow[0,1],
$$

provides the degree in which the $i^{\text {th }}$ fuzzy goal is attained. By using the concept of fuzzy decision, "max-min" of Bellman and Zadeh [12] and by introducing the auxiliary variable $\lambda$, a fuzzy goal programming problem adopt the formulation in $[7]$ :

\section{$\operatorname{Max} \lambda$}

Subject to

$$
\begin{gathered}
\lambda \leq \mu_{i}\left(z_{i}(x)\right), \quad i=1,2, \ldots, k \\
x \in F, \quad x \geq 0, \lambda \in[0,1] .
\end{gathered}
$$

Let us suppose that the membership function $\mu_{i}\left(z_{i}(x)\right),(i=1,2, \ldots, k)$ is piecewise linear shaped increasing function, as shown in Fig. 4.1

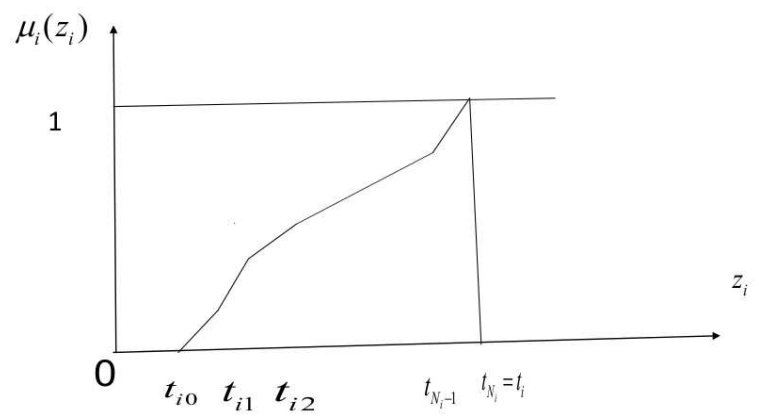

Fig.4.1 
Then, the membership function is constructed as follows:

$$
\mu_{i}\left(z_{i}(x)\right)=\sum_{j=0}^{N_{i}} \alpha_{i j}\left|z_{i}(x)-t_{i j}\right|+\mu_{i}\left(t_{i j}\right), \quad j=0,1, \ldots, N_{i},
$$

where $\alpha_{i j}=\left(\mu_{i}\left(t_{i j+1}\right)-\mu_{i}\left(t_{i j}\right)\right) /\left(t_{i j+1}-t_{i j}\right)$ is the slope of the line segment originated from $t_{i j}$ and terminated at $t_{i j+1}$ and $\mu_{i}\left(t_{i j}\right)$ is the membership value at $z_{i}=t_{i j}$. To formulate model (4.2) as a linear programming problem, following the concepts and procedures as in classical GP, we introduce the nonnegative deviational variables $n_{i j}, p_{i j}$ :

$$
z_{i}(x)+n_{i j}-p_{i j}=t_{i j}
$$

$j=0,1, \ldots, N_{i}, i=1,2, \ldots, k$.

Thus,each piecewise linear membership function $\mu_{i}\left(z_{i}(x)\right)$ can be expressed as

$$
\mu_{(}\left(z_{i}(x)\right)=\sum_{j=0}^{N_{i}} \alpha_{i j}\left(n_{i j}+p_{i j}\right)+\mu_{i}\left(t_{i j}\right) .
$$

Then problem (4.2) can be converted into the following problem: Max $\lambda$ Subject to

$$
\begin{gathered}
\lambda \leq \sum_{j=0}^{N_{i}} \alpha_{i j}\left(n_{i j}+p_{i j}\right)+\mu_{i}\left(t_{i j}\right), \\
z_{i}(x)+n_{i j}-p_{i j}=t_{i j}, \\
x \in F, \quad x \geq 0, \quad \lambda \in[0,1],
\end{gathered}
$$

$n_{i j} \geq 0, p_{i j} \geq 0, i=1,2, \ldots, k, j=0,1,2, \ldots, N_{i}$.

The solution of problem (4.6) will give the solution of the GP problem, (3.1) under fuzzy environment.

Example. Consider the GP problem

$$
\begin{aligned}
& \operatorname{goal}\left\{x_{1}=z_{1}\right\}, z_{1} \geq 4, \\
& \operatorname{goal}\left\{x_{2}=z_{2}\right\}, z_{2} \geq 2, \\
& \operatorname{goal}\left\{x_{3}=z_{3}\right\}, z_{3} \geq 3,
\end{aligned}
$$

s.t.

$$
\begin{gathered}
2 x_{1}+3 x_{2}+2 x_{3} \leq 4, \\
x_{1}+2 x_{2} \leq 5
\end{gathered}
$$




$$
x_{1}, x_{2}, x_{3} \geq 0
$$

Without lost of generality we define the membership values of the piecewise membership function of each goal as follows:

$$
\begin{aligned}
& \mu_{1}\left(z_{1}\right): 0 \quad \begin{array}{llll}
0 & .5 & .8 & 1,
\end{array} \\
& z_{1}: 123 \quad 3 \quad 4 \text {, }
\end{aligned}
$$

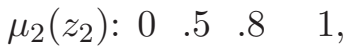

$$
\begin{aligned}
& z_{2}: \begin{array}{llll}
.5 & 1 & 1.5 & 2,
\end{array} \\
& \mu_{3}\left(z_{3}\right): 0 \quad 0 \quad .5 \quad .8 \quad 1 \text {, } \\
& z_{3}: 1 \quad 1.5 \quad 2 \quad 3 .
\end{aligned}
$$

Calculate $\alpha_{i j}$ as follows: $\alpha_{10}=0.5, \alpha_{11}=0.3, \alpha_{12}=0.2, \alpha_{20}=1, \alpha_{21}=0.6$, $\alpha_{22}=0.4, \alpha_{30}=1, \alpha_{31}=0.6, \alpha_{32}=0.2$.

We obtain: $\mu_{1}\left(z_{1}\right)=z_{1}+0.8, \mu_{2}\left(z_{2}\right)=2 z_{2}-0.4, \mu_{3}\left(z_{3}\right)=1.8 z_{3}-1$.

The equivalent LP problem is obtained as $\operatorname{Max} \lambda$

$$
\begin{aligned}
\text { s.t. } \lambda \leq\left(n_{11}+p_{11}\right)+0.8 & \\
\lambda \leq 2\left(n_{21}+p_{21}\right)-0.4 & \\
\lambda \leq\left(n_{31}+p_{31}\right)-1 & \\
x_{1}+n_{11}-p_{11} & =4 \\
x_{2}+n_{21}-p_{21} & =2 \\
x_{3}+n_{31}-p_{31} & =3 \\
2 x_{1}+3 x_{2}+2 x_{3} & \leq 4 \\
x_{1}+2 x_{2} & \leq 5 \\
x_{1}, x_{2}, x_{3}, n_{11}, n_{21}, n_{31}, p_{11}, p_{21}, p_{31} & \geq 0, \lambda \in[0,1]
\end{aligned}
$$

By using TORA software, after 13 iterations, the solution is obtained as $x_{1}=0, x_{2}=0.07, x_{3}=1.87, \lambda=1$.

\section{Conclusion}

The proposed method will provide a new strategy for solving FGP problems with piecewise linear membership functions. 


\section{Acknowledgments}

The authors are hightly appreciated to the referees for giving suggestions in the improvement of the paper. And also, the authors are much thankful to the UGC for financial assistance under RGNF scheme.

\section{References}

[1] A. Charnes, W.W. Cooper, Management Models and Industrial Application of Linear Programming, Wiley, New York (1961).

[2] A. Charnes, W.W. Cooper, Goal programming and multipleobjective optimisations (Part I), European Journal of Operational Research, 1 (1977), $39-54$.

[3] C. Romero, Handbook of Critical Issues in Goal Programming, Oxford, New York, Pergamon Press (1991).

[4] C. Romero, A general structure of achievement function for a goal programming model, European Journal of Operational Research, 153 (2004), 675-686.

[5] E.K. Can, M. Houk, Real-time reservoir operation by goal programming, Journal Water Resources Planning and Management, 110 (1984), 297-309.

[6] E.L. Hannan, Linear programming with multiple fuzzy goals, Fuzzy Sets and Systems, 6 (1981), 235-248.

[7] H.J. Zimmermann, Fuzzy Set Theory and its Applications, 2-nd Edition, Kluwer Academic Publishers, Boston-Dordrecht-London (1991), 247-248.

[8] J.P. Ignizio, Goal Programming and Extensions, Lexington Books, Lexington, MA (1976).

[9] J.S. Dyer, On the relationship between goal programming and multiattribute utility theory, Discussion Paper, No. 69, Management Science Study Centre, University of California, LA (1977).

[10] M.H. Lohgaonkar, V.H. Bajaj, V.A. Jadhav, Additive fuzzy multiple goal programming model for unbalanced multi-objective transportation problem, International Journal of Machine Intelligence (2010), 29-34. 
[11] R.B. Flavell, A new goal programming formulation. Omega, The International Journal of Management Science, 4 (1976), 731-732.

[12] R.E. Bellman, L.A. Zadeh, Decision making in a fuzzy environment, Management Science, 17 (1970), 141-164.

[13] R. Narasimhan, Goal programming in fuzzy environment, Decision Sciences, 11 (1980), 325-336.

[14] R.N. Tiwari, S. Dharma, J.R. Rao, Priority structure in fuzzy goal programming, Fuzzy Sets and Systems (1985), 251-259.

[15] R.N. Tiwari, S. Dharma, J.R. Rao, An additive model, Fuzzy Sets and Systems, 24 (1987), 27-34.

[16] S.M. Lee, Goal Programming for Decision Analysis, Auerbach Publishers, Philadelphia (1972).

[17] W. F. Abd El-Wahed, Sang M. Lee, Interactive fuzzy goal programming for multi-objective transportation problems, The nternational Journal of Management Science (2006), 158-166. 